\title{
ANALYSIS OF SURFACE LEACHING PROCESSES IN VITRIFIED HIGH-LEVEL NUCLEAR WASTES USING IN-SITU RAMAN IMAGING AND ATOMISTIC MODELLING
}

\author{
by \\ Joseph H. Simmons and David E. Clark \\ University of Florida \\ Gainesville, FL 32611 \\ Grant Number: DE-FG07-96ER-45616
}

First Year Progress Report

\section{Objectives}

(1) To investigate the development of Raman spectroscopy for remote, in-situ, realtime measurement of the processes underlying chemical corrosion of glasses,

(2) To conduct Raman spectroscopy measurements and quantum mechanical modelling studies of the transition states, corrosion products, and transition state energies for the hydrate species of higher valence and multivalent ions formed in the reconstructed glass surface.

(3) To use these results to model long-term corrosion behavior of complex borosilicate wasteform glasses.

(3) To apply the Raman spectroscopy and modelling methods developed here for the remote analysis of leaching processes in waste glasses containing radioactive components, and for imaging of variations in leaching behavior due to composition inhomogeneities in large scale waste glass products.

\section{Results of First Year Research}

During the first year, we primarily addressed Objective (1) which is to develop a methodology for the remote monitoring of leaching processes in glasses by Raman spectroscopy. We assembled a micro and macro Raman system for examining surface structure in glass samples, in-situ within the leaching vessel. The Raman spectrometer was prepared for imaging by installing a CCD detector which gives 2-dimensional information. The latter can be used to obtain spectrographic data in one dimension and to scan variations in materials behavior across the other dimension. By scanning the sample in a perpendicular direction, it is possible to conduct 2-dimensional spectral 
analysis of the sample surface. The plan is to select one or several identifying Raman lines that follow the leaching process After that, it will be possible to introduce a slit configuration and use both dimensions of the CCD detector for scanning the sample surface while examining only the selected spectral feature.

Glass samples consisting of alkali silicates were first examined. The samples were melted in a conventional electric. furnace in $100 \mathrm{gr}$ batches and poured into brass molds. The samples were cut and polished under water-free conditions and then immersed under water in petri dishes. By leaving the upper surface uncovered, we focused the objective lens of a microscope on the submerged glass surface. Light from an Argon laser operating at $514.5 \mathrm{~nm}$ with approximately $100 \mathrm{~mW}$ of power was directed to the glass surface at a large angle of incidence $\left(55^{\circ}\right)$.

Raman spectra were collected in a $0.6 \mathrm{~m}$ spectrometer with a 1200 line $/ \mathrm{mm}$ grating using a supernotch filter to block the laser line. Typical data are shown in the attached figure. Samples of the following compositions were tested: $20 \% \mathrm{Na}_{2} \mathrm{O}-80 \% \mathrm{SiO}_{2}$ and $30 \% \mathrm{Li}_{2} \mathrm{O}-70 \% \mathrm{SiO}_{2}$. The attached figure reports the results from the $\mathrm{Li}_{2} \mathrm{O}-\mathrm{SiO}_{2}$ glass. The results show a significant difference between the unleached and water-exposed samples. This is evident in the relative amplitudes of the Raman peaks at $436 \mathrm{~cm}^{-1}$ and $582 \mathrm{~cm}^{-1}$. These 2 peaks are associated with vibrations of the alkali coordinated nonbridging oxygen. In fact, the ratio of the 582 to the 436 peaks gives a measure of the alkali content of the material. By examining the sample surface only, and conducting the tests while the sample is submerged, we observed a decrease in the $582 \mathrm{~cm}^{-1}$ peak and an increase in the $436 \mathrm{~cm}^{-1}$ peak with increased exposure of the sample to the aqueous environment. This is shown in the attached figure and is clear indication of the progressive dealkalization of the glass surface by water. The peaks can be fitted to Gaussian shapes and the integrated intensity will yield a direct measure of the alkali content of the glass surface. In addition, the angle of incidence of the laser beam can be adjusted to provide more penetration into the sample and yield composition profiles.

\section{Significance of results}

The results are the first demonstration of leached surface analysis in a submerged sample by any method. The Raman method tends itself welt to a quantitative measure of the leached surface composition in-situ, without removing the sample from its bath. This approach has the promise of conducting surface analysis measurements remotely on glasses that are either fully submerged or under various storage conditions. In addition, because the sample does not need to be removed from the bath, this method has the potential for examination of radioactive materials remotely and without contamination of measuring equipment. Finally, all other analytical methods require the sample to be removed from the bath thus causing irreversible damage to the corroded surface by the need for coatings or just simply drying the surface and causing cracks to develop. The Raman technique developed is non-intrusive and does not damage the sample, thus tests can be continued indefinitely on the same sample. 


\section{Studies for next year}

We are melting borosilicate glasses of the waste-glass composition to investigate the sensitivity of the method for other glass components. Tests will also be conducted under high surface-to-volume conditions in order to form precipitates on the glass surface. Raman spectroscopy has a high potential for the detection and identification of the precipitates. We are also in discussions with research staff at PNL Laboratories to transfer our methodology to hot cell experiments to be conducted next year (Objective 4). Raman tests on simulated non-radioactive samples will be supported by FTIR and XPS measurements to determine leached surface composition by other methods. These methods, however, require drying and coating the samples, respectively.

Modelling studies of the transition states (Objective 2) during leaching will be enabled by the surface Raman studies. Modelling calculations using the molecular orbital theory approach will be conducted next year.

\section{Students supported}

Steve MacDonald - graduate student (MS candidate in the Dept. of Materials Science)

Greg Darby - graduate student (MS candidate)

Heather Mockler and Eileen Gonzalez - undergraduate students 

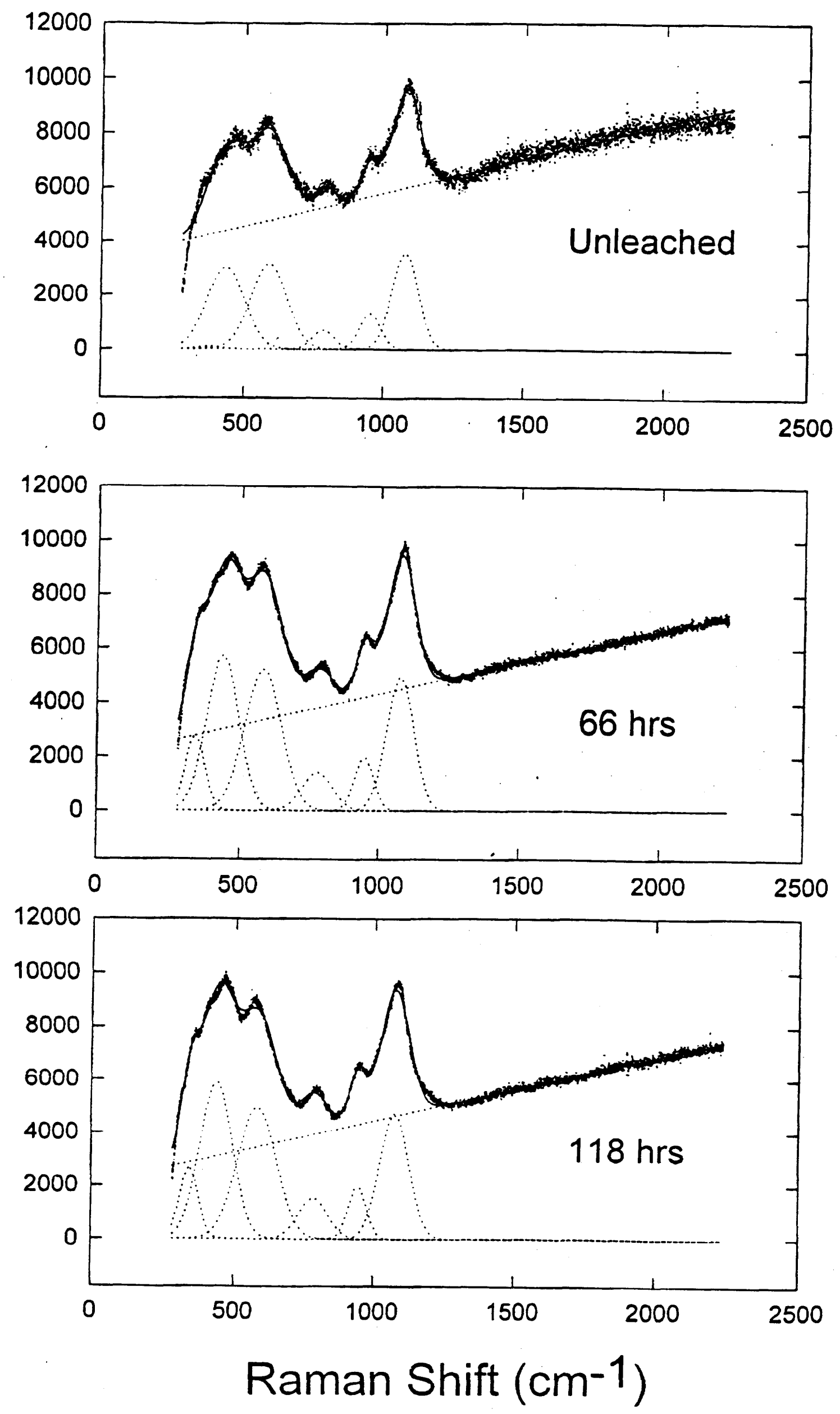